TRANSACTIONS OF THE

AMERICAN MATHEMATICAL SOCIETY

Volume 355, Number 5, Pages 1773-1786

S 0002-9947(03)03185-4

Article electronically published on January 13, 2003

\title{
CASTELNUOVO-MUMFORD REGULARITY AND EXTENDED DEGREE
}

\author{
MARIA EVELINA ROSSI, NGÔ VIÊT TRUNG, AND GIUSEPPE VALLA
}

\begin{abstract}
Our main result shows that the Castelnuovo-Mumford regularity of the tangent cone of a local ring $A$ is effectively bounded by the dimension and any extended degree of $A$. From this it follows that there are only a finite number of Hilbert-Samuel functions of local rings with given dimension and extended degree.
\end{abstract}

\section{INTRODUCTION}

The Castelnuovo-Mumford regularity is a kind of universal bound for important invariants of graded algebras, such as the maximum degree of the syzygies and the maximum non-vanishing degree of the local cohomology modules (see Section 1). It has been used as a measure for the complexity of computational problems in algebraic geometry and commutative algebra (see e.g. [EG], [BM], [V2]). One has often tried to find upper bounds for the Castelnuovo-Mumford regularity in terms of simpler invariants. The simplest invariants which reflect the complexity of a graded algebra are the dimension and the multiplicity. However, the Castelnuovo-Mumford regularity cannot be bounded in terms of the multiplicity and the dimension.

Extended degree was recently introduced by Vasconcelos et al. [DGV], [V1, V2] in order to capture the size of a module along with some of the complexity of its structure. It is a numerical function on the category of finitely generated modules over local or graded rings which generalizes the usual notion of multiplicity and degree (see Section 2). These invariants tend to have a homological character, but are still amenable to explicit computation by computer algebra systems. It has been shown by Doering, Gunston and Vasconcelos [DGV] that the Castelnuovo-Mumford regularity of a graded algebra is less than any extended degree.

In this paper we show that the Castelnuovo-Mumford regularity of the tangent cone of a local ring is bounded above by an exponential function of the dimension and any extended degree. This bound is neither a consequence nor a simple generalization of the result of [DGV], since there is no relationship between the extended degrees of a local ring and those of its tangent cone.

As an application we give upper bounds for the coefficients of the Hilbert-Samuel function in terms of any extended degree. It follows that there are only a finite number of Hilbert-Samuel functions for local rings with given dimension and extended degree. This application covers three recent results on the finiteness of Hilbert

Received by the editors August 9, 2002.

2000 Mathematics Subject Classification. Primary 13A30, 13D45.

The first and third authors are partially supported by MPI of Italy. The second author is partially supported by the National Basic Research Program of Vietnam. 
functions. The first result is due to Srinivas and Trivedi [ST2, who showed that there exist only a finite number of Hilbert functions of Cohen-Macaulay local rings with given dimension and multiplicity (see also [K1], ST1], [Tri1]). This result was extended by Trivedi [Tri2] to the class of generalized Cohen-Macaulay rings by involving the lengths of local cohomology modules. The last result is due to Rossi, Valla and Vasconcelos [RVV], who showed that there exist only a finite number of Hilbert functions of graded algebras with given dimension and extended degree.

Let $(A, \mathfrak{m})$ be a local ring with infinite residue field, and $d=\operatorname{dim} A$. We will denote by $\operatorname{reg}(G)$ the Castelnuovo-Mumford regularity of the associated graded ring $G=\bigoplus_{n \geq 0} \mathfrak{m}^{n} / \mathfrak{m}^{n+1}$, and we will write the Hilbert-Samuel polynomial of $A$ in the form

$$
P_{A}(X):=\sum_{i=0}^{d}(-1)^{i} e_{i}(A)\left(\begin{array}{c}
X+d-i \\
d-i
\end{array}\right) .
$$

By definition, $P_{A}(n)=\ell\left(A / \mathfrak{m}^{n+1}\right)$ for $n \gg 0$. Moreover, we set $e(A)=e_{0}(A)$ and $I(A)=D(A)-e(A)$, where $D(A)$ is a given extended degree of $A$. We always have $I(A) \geq 0$, with $I(A)=0$ if and only if $A$ is a Cohen-Macaulay ring.

Our main results can be formulated as follows.

Theorem 3.3, Let $D(A)$ be an arbitrary extended degree of $A$. Then

(i) $\operatorname{reg}(G) \leq e(A)+I(A)-1$ if $d=1$,

(ii) $\operatorname{reg}(G) \leq e(A)^{(d-1) !-1}\left[e(A)^{2}+e(A) I(A)+2 I(A)-e(A)\right]^{(d-1) !}-I(A)$ if $d \geq 2$.

Theorem 4.1, Let $D(A)$ be an arbitrary extended degree of $A$. Then

(i) $\left|e_{1}(A)\right| \leq \frac{e(A)[e(A)-1]}{2}+I(A)$,

(ii) $\left|e_{i}(A)\right| \leq e(A)^{i !-i}\left[e(A)^{2}+e(A) I(A)+2 I(A)\right]^{i !}-1$ if $i \geq 2$.

Although these bounds are far from being optimal, they provide the means to make a priori estimates in local algebra, following the philosophy of [DGV] and V2.

The starting point of our approach is the observation that the regularity of the tangent cone can be estimated by means of the geometric regularity (Propositions 3.1 and 3.2). Due to a result of Mumford [Mu], the problem of bounding the geometric regularity can be reduced to the problem of bounding the Hilbert polynomial (Theorem 1.4). This idea has been used in [ST2] and [Tri2]. But unlike [ST2] and Tri2], which refer to deep results from algebraic geometry such as Grothendieck's formal function theorem, we use standard algebraic methods to solve this problem. The key point is a uniform bound for the Hilbert-Samuel function in terms of any extended degree (Theorem 2.1). By induction, this bound allows us to estimate the regularity of the tangent cone. The coefficients of the Hilbert-Samuel polynomial can be bounded then by a device due to Vasconcelos.

We would like to mention that Trivedi Tri1], Tri2] has dealt with the Hilbert function of Cohen-Macaulay and generalized Cohen-Macaulay modules with respect to $\mathfrak{m}$-primary ideals. Our approach shows that the results of this paper can also be extended to this general situation. But it is not our intention to go so far.

The paper is organized as follows. In Section 1 we prepare some facts and results on the regularity of graded algebras. In Section 2 we estimate the geometric regularity in terms of any extended degree. In Section 3 and Section 4 we prove 
the bound for the regularity of the tangent cone and the bounds for the coefficients of the Hilbert-Samuel polynomial, respectively.

\section{Regularity of GRAded Algebras}

Throughout this section let $S=k\left[x_{1}, \ldots, x_{r}\right]$ be a polynomial ring over a field $k$. Let $M$ be a finitely generated graded $S$-module. Let

$$
0 \longrightarrow F_{s} \longrightarrow \cdots \longrightarrow F_{1} \longrightarrow F_{0} \longrightarrow M \longrightarrow 0
$$

be a minimal graded free resolution of $M$. Write $b_{i}$ for the maximum of the degrees of the generators of $F_{i}$. Following [E, Section 20.5], we say that $M$ is $m$-regular for some integer $m$ if $b_{j}-j \leq m$ for all $j$. The Castelnuovo-Mumford regularity $\operatorname{reg}(M)$ of $M$ is defined to be the least integer $m$ for which $M$ is $m$-regular, that is,

$$
\operatorname{reg}(M)=\max \left\{b_{i}-i \mid i=0, \ldots, s\right\} .
$$

It is well known that $M$ is $m$-regular if and only if $\operatorname{Ext}_{S}^{i}(M, S)_{n}=0$ for all $i$ and all $n \leq-m-i-1$ (see [EG] and [E, Proposition 20.16]). This result is hard to apply, because in principle infinitely many conditions must be checked. However, in some cases, it suffices to check just a few.

We say that $M$ is weakly m-regular if $\operatorname{Ext}_{S}^{i}(M, S)_{-m-i-1}=0$ for all $i$ E, Section 20.5]. Concerning this weaker notion of regularity, we have the following result of Mumford.

Theorem 1.1 (see [E, Theorem 20.17]). If $M$ is weakly m-regular and $L$ is the maximal submodule of $M$ having finite length, then $M / L$ is $m$-regular.

If $M$ has positive depth, then $L=0$, so that $m$-regularity and weak $m$-regularity coincide. It is less known that this is also the case for homogeneous quotient rings of $S$ (not necessarily of positive depth).

Corollary 1.2. Let $R=S / I$, where $I$ is a homogeneous ideal. If $R$ is weakly $m$-regular, then $R$ is m-regular.

Proof. By Theorem[1.1, $R / L$ is $m$-regular, where $L$ is the largest ideal of $R$ of finite length. Hence $m \geq 0$ by the definition of regularity. From the short exact sequence

$$
0 \longrightarrow I \longrightarrow S \longrightarrow R \longrightarrow 0
$$

we get

$$
\begin{aligned}
\operatorname{Ext}_{S}^{0}(I, S)_{-m-1} & \simeq \operatorname{Ext}_{S}^{1}(R, S)_{-m-1}=0, \\
\operatorname{Ext}_{S}^{i}(I, S)_{-m-i-1} \simeq \operatorname{Ext}_{S}^{i+1}(R, S)_{-m-i-1} & =0, i \geq 1,
\end{aligned}
$$

so that $I$ is weakly $(m+1)$-regular. Since $I$ has positive depth, $I$ is $(m+1)$ regular by Theorem 1.1. Looking at the minimal graded free resolution of $I$, we can conclude that $R$ is $m$-regular.

Let $R$ be a standard graded algebra over a field $k$. Let $M$ now be a finitely generated graded $R$-module. For any integer $i$ we denote by $H_{R_{+}}^{i}(M)$ the $i$ th local cohomology module of $M$, where $R_{+}$is the maximal graded ideal of $R$.

If $R=S / I$, where $I$ is a homogeneous ideal, then $H_{R_{+}}^{i}(M)=H_{S_{+}}^{i}(M)$. By local duality (see [E, A4.2]) we have

$$
H_{S_{+}}^{i}(M)_{m} \cong \operatorname{Ext}_{S}^{r-i}(M, S)_{-m-r}
$$


for all $i$ and $m$. Thus, $M$ is $m$-regular if and only if $H_{R_{+}}^{i}(M)_{n}=0$ for all $i$ and $n \geq m-i+1$, and $M$ is weakly $m$-regular if and only if $H_{R_{+}}^{i}(M)_{m-i+1}=0$ for all $i$. In particular, $\operatorname{reg}(M)$ is the least integer $m$ for which $H_{R_{+}}^{i}(M)_{n}=0$ for all $i$ and $n \geq m-i+1$. Hence the Castelnuovo-Mumford regularity can be defined for any finite $R$-module, regardless of its presentation.

We can use the Castelnuovo-Mumford regularity to control the behavior of the Hilbert function. Let $h_{M}(n)$ be the Hilbert function of $M$, and $p_{M}(n)$ the Hilbert polynomial of $M$, which is by definition the unique polynomial in $\mathbf{Q}[X]$ for which $p_{M}(n)=h_{M}(n)$ for all $n \gg 0$. Their difference is determined by the following classical formula of Serre (see e.g. [BH, Theorem 4.4.3]):

$$
h_{M}(n)-p_{M}(n)=\sum_{i \geq 0}(-1)^{i} \operatorname{dim}_{k} H_{R_{+}}^{i}(M)_{n} .
$$

Hence we immediately obtain the following consequence.

Lemma 1.3. $h_{R}(n)=p_{R}(n)$ for $n>\operatorname{reg}(R)$.

The regularity in algebraic geometry is defined a bit differently. Let $\mathcal{F}$ be a coherent sheaf on $\mathbb{P}^{r}$. Then $\mathcal{F}$ is called $m$-regular if $H^{i}\left(\mathbb{P}^{r}, \mathcal{F}(m-i)\right)=0$ for all $i>0$, where $H^{i}\left(\mathbb{P}^{r}, \mathcal{F}\right)$ denotes the $i$ th sheaf cohomology of $\mathcal{F}[\mathrm{Mu}$, p. 99, Definition]. This regularity is related to the Castelnuovo-Mumford regularity by the Serre-Grothendieck correspondence, which says that if $\mathcal{F}$ is the sheaf associated to the $R$-module $M$, then $H^{i}\left(\mathbb{P}^{r}, \mathcal{F}(n)\right) \cong H_{R_{+}}^{i+1}(M)_{n}$ for all $n$ and $i \geq 1$.

Due to a result of Mumford in $\overline{\mathrm{Mu}}$, the regularity of an ideal sheaf can be estimated in terms of that of a generic hyperplane section by means of the Hilbert polynomial. We present here an algebraic version of this result, for which we shall need the following notation.

Definition. We say that $M$ is geometrically m-regular if $H_{R_{+}}^{i}(M)_{n}=0$ for all $i>0$ and $n \geq m-i+1$, and we define the geometric regularity g-reg $(M)$ of $M$ to be the least integer $m$ for which $M$ is geometrically $m$-regular.

We always have $\mathrm{g}$-reg $(M) \leq \operatorname{reg}(M)$ and $\mathrm{g}-\operatorname{reg}(M)=\operatorname{reg}(M)$ if $\operatorname{depth} M>0$.

Following [Tru2], we call a homogeneous element $z$ of $R$ filter-regular if $z \notin Q$ for any relevant associated prime ideal $Q$ of $R$. Filter-regular linear forms always exist if $k$ is an infinite field.

The following theorem follows from the proof of $[\mathrm{Mu}$, p. 101, Theorem]. We insert here a proof for completeness. Note that $R=S / I$ is geometrically $n$-regular if and only if the sheafification of $I$ is $(n+1)$-regular.

Theorem 1.4. Let $R$ be a standard graded algebra with $\operatorname{dim} R \geq 1$. Let $z$ be a filter-regular linear form of $R$. If $R / z R$ is geometrically $m$-regular, then $R$ is geometrically $\left(m+p_{R}(m)-h_{R / L}(m)\right)$-regular, where $L$ denotes the largest ideal of finite length of $R$.

Proof. Since $H_{R_{+}}^{i}(L)=0$ for $i>0$, we get $H_{R_{+}}^{i}(R) \cong H_{R_{+}}^{i}(R / L)$ for $i>0$. Hence $\mathrm{g}$-reg $(R)=\mathrm{g}-\operatorname{reg}(R / L)$. Similarly, since $L+z R / z R$ is an ideal of finite length in $R / z R$, we also have

$$
\mathrm{g}-\operatorname{reg}(R / z R)=\mathrm{g}-\operatorname{reg}((R / z R) / L(R / z R))=\mathrm{g}-\operatorname{reg}((R / L) / z(R / L)) .
$$

Further, it is clear that $p_{R}(X)=p_{R / L}(X)$. Thus, replacing $R / L$ by $R$, we may assume that $z$ is a regular element in $R$. With this assumption we have g-reg $(R)=$ 
$\operatorname{reg}(R)=\operatorname{reg}(R / z R)$. We need to show that if $R / z R$ is geometrically $m$-regular, then $R / z R$ is $\left(m+p_{R}(m)-h_{R}(m)\right)$-regular.

Since $R / z R$ is geometrically $m$-regular, we have $H^{i}(R / z R)_{n-i+1}=0$ for every $n \geq m, i>0$. If we can prove $H_{R_{+}}^{0}(R / z R)_{n+1}=0$ for some $n \geq m$, then $R / z R$ is weakly $n$-regular, hence $n$-regular by Corollary 1.2. So it is sufficient to show that if $R / z R$ is geometrically $m$-regular, there exists an integer $j$ with $m+1 \leq j \leq m+p_{R}(m)-h_{R}(m)+1$ such that $H_{R_{+}}^{0}(R / z R)_{j}=0$.

From the short exact sequence $0 \longrightarrow R(-1) \stackrel{z}{\longrightarrow} R \longrightarrow R / z R \longrightarrow 0$ we get a long exact sequence of local cohomology modules:

$$
\begin{aligned}
0 \longrightarrow & H_{R_{+}}^{0}(R / z R)_{n} \longrightarrow H_{R_{+}}^{1}(R)_{n-1} \longrightarrow H_{R_{+}}^{1}(R)_{n} \longrightarrow H_{R_{+}}^{1}(R / z R)_{n} \\
& \longrightarrow \cdots \longrightarrow H_{R_{+}}^{i}(R / z R)_{n} \longrightarrow H_{R_{+}}^{i+1}(R)_{n-1} \longrightarrow H_{R_{+}}^{i+1}(R)_{n} \longrightarrow \cdots
\end{aligned}
$$

for every integer $n$. Since $R / z R$ is geometrically $m$-regular, we have $H_{R_{+}}^{i}(R / z R)_{n}=$ 0 for ever $i>0$ and $n \geq m-i+1$. Putting this into the above exact sequence yields, with the assumption $n \geq m$,

$$
\operatorname{dim}_{k} H_{R_{+}}^{1}(R)_{n-1}-\operatorname{dim}_{k} H_{R_{+}}^{1}(R)_{n}=\operatorname{dim}_{k} H_{R_{+}}^{0}(R / z R)_{n}
$$

and the injectivity of the map $H_{R_{+}}^{i+1}(R)_{n-1} \longrightarrow H_{R_{+}}^{i+1}(R)_{n}$ for $i \geq 1$. Because $H_{R_{+}}^{i+1}(R)_{t}=0$ for $t \gg 0$, it follows that $H_{R_{+}}^{i+1}(R)_{t}=0$ for $i \geq 1$ and $t \geq m-1$.

Since $H_{R_{+}}^{0}(R)=0$, this implies

$$
h_{R}(m)-p_{R}(m)=\sum_{i \geq 0}(-1)^{i} \operatorname{dim}_{k} H_{R_{+}}^{i}(R)_{m}=-\operatorname{dim}_{k} H_{R_{+}}^{1}(R)_{m} .
$$

Put $s=1+m+p_{R}(m)-h_{R}(m)=1+m+\operatorname{dim}_{k} H_{R_{+}}^{1}(R)_{m}$ and assume for a contradiction that $H_{R_{+}}^{0}(R / z R)_{j} \neq 0$ for every integer $j$ with $m+1 \leq j \leq s$. By (1) we get

$$
\begin{aligned}
\operatorname{dim}_{k} H_{R_{+}}^{1}(R)_{m} & =\sum_{j=m+1}^{s} \operatorname{dim}_{k} H_{R_{+}}^{0}(R / z R)_{j}+\operatorname{dim}_{k} H_{R_{+}}^{1}(R)_{s} \\
& \geq s-m>\operatorname{dim}_{k} H_{R_{+}}^{1}(R)_{m} .
\end{aligned}
$$

The proof of Theorem 1.4 is now complete.

\section{EXtended DEGREe VERSus GEOMETRIC REGULARITY}

Let $(A, \mathfrak{m})$ be a local ring with infinite residue field. Let $\mathcal{M}(A)$ denote the class of finitely generated $A$-modules. Following [DGV] and [V2], an extended degree (or cohomological degree) on $\mathcal{M}(A)$ is a numerical function $D(\cdot)$ on $\mathcal{M}(A)$ such that the following properties hold for every module $M \in \mathcal{M}(A)$ :

(i) $D(M)=D(M / L)+\ell(L)$, where $L$ is the maximal submodule of $M$ having finite length,

(ii) $D(M) \geq D(M / x M)$ for a generic element $x$ of $\mathfrak{m}$, and

(iii) $D(M)=e(M)$ if $M$ is a Cohen-Macaulay $A$-module, where $e(M)$ denotes the multiplicity of $M$ with respect to $\mathfrak{m}$.

Example. The prototype of an extended degree is the homological degree hdeg $(M)$ introduced and studied by Vasconcelos in V1] (see also [V2]). 
If $A$ is a homomorphic image of a Gorenstein $\operatorname{ring} S$ with $\operatorname{dim} S=n$ and $M \in$ $\mathcal{M}(A)$ with $\operatorname{dim} M=r$, we define

$$
\operatorname{hdeg}(M):=e(M)+\sum_{i=0}^{r-1}\left(\begin{array}{c}
r-1 \\
i
\end{array}\right) \operatorname{hdeg}\left(\operatorname{Ext}_{S}^{n-i}(M, S)\right) .
$$

This definition is recursive on the dimension, since $\operatorname{dim}_{\operatorname{Ext}_{S}^{n-i}}^{n-}(M, S)<r$ for $i=$ $0, \ldots, r-1$.

If $A$ is not a homomorphic image of a Gorenstein ring, we only need to put

$$
\operatorname{hdeg}(M):=\operatorname{hdeg}\left(M \otimes_{A} \hat{A}\right),
$$

where $\hat{A}$ denotes the $\mathfrak{m}$-adic completion of $A$.

In particular, if $M$ is a generalized Cohen-Macaulay module, that is, $\ell\left(H_{\mathfrak{m}}^{i}(M)\right)<$ $\infty$ for $i<r=\operatorname{dim} M$, where $H_{\mathfrak{m}}^{i}(M)$ denotes the $i$ th local cohomology module of $M$ with support $\mathfrak{m}$, then

$$
\operatorname{hdeg}(M)=e(M)+\sum_{i=0}^{r-1}\left(\begin{array}{c}
r-1 \\
i
\end{array}\right) \ell\left(H_{\mathfrak{m}}^{i}(M)\right) .
$$

This class of modules is rather large. In fact, if $A$ is a quotient ring of a CohenMacaulay ring, then $M$ is a generalized Cohen-Macaulay module if and only if $M$ is locally Cohen-Macaulay on the punctured spectrum of $A$ and $\operatorname{Supp}(M)$ is equidimensional.

Any extended degree $D(M)$ will satisfy $D(M) \geq e(M)$, with equality holding if and only if $M$ is a Cohen-Macaulay module. Following [DGV], we call the difference

$$
I(M):=D(M)-e(M)
$$

a Cohen-Macaulay deviation of $M$. It is obvious that $I(M)$ satisfies the following conditions:

(i') $I(M)=I(M / L)+\ell(L)$,

$\left(\mathrm{ii}^{\prime \prime}\right) I(M) \geq I(M / x M)$ for a generic element $x$ of $\mathfrak{m}$.

The Hilbert-Samuel function can be bounded in terms of any extended degree as follows.

Theorem 2.1. Assume that $d=\operatorname{dim} A \geq 1$. Let $I(A)$ be an arbitrary CohenMacaulay deviation of $A$. For all $n \geq 0$ we have

$$
\ell\left(A / \mathfrak{m}^{n+1}\right) \leq e(A)\left(\begin{array}{c}
n+d-1 \\
d
\end{array}\right)+I(A)\left(\begin{array}{c}
n+d-2 \\
d-1
\end{array}\right)+\left(\begin{array}{c}
n+d-1 \\
d-1
\end{array}\right)
$$

Proof. Let $x_{1}, \ldots, x_{d}$ be a system of generic elements of $\mathfrak{m}$ such that $e(A)$ is the multiplicity of $A$ with respect to the ideal $\mathfrak{q}=\left(x_{1}, \ldots, x_{d}\right)$. We have

$$
\ell\left(A / \mathfrak{m}^{n+1}\right) \leq \ell\left(A / \mathfrak{m} \mathfrak{q}^{n}\right)=\ell\left(A / \mathfrak{q}^{n}\right)+\ell\left(\mathfrak{q}^{n} / \mathfrak{m} \mathfrak{q}^{n}\right) .
$$

Since $x_{1}, \ldots, x_{d}$ are analytically independent,

$$
\ell\left(\mathfrak{q}^{n} / \mathfrak{m} \mathfrak{q}^{n}\right)=\left(\begin{array}{c}
n+d-1 \\
d-1
\end{array}\right) .
$$

It remains to show that

$$
\ell\left(A / \mathfrak{q}^{n}\right) \leq e(A)\left(\begin{array}{c}
n+d-1 \\
d
\end{array}\right)+I(A)\left(\begin{array}{c}
n+d-2 \\
d-1
\end{array}\right) .
$$


If $d=1, A / L$ is a Cohen-Macaulay ring, where $L$ is the largest ideal of finite length. Hence $\ell\left(A / \mathfrak{q}^{n}+L\right)=e(A) n$ and $I(A)=\ell(L)$. From this it follows that

$$
\ell\left(A / \mathfrak{q}^{n}\right) \leq \ell\left(A / \mathfrak{q}^{n}+L\right)+\ell(L)=e(A) n+I(A) .
$$

If $d>1$, we put $\bar{A}=A /\left(x_{1}\right)$ and $\overline{\mathfrak{q}}=\mathfrak{q} /\left(x_{1}\right)$. Then $\operatorname{dim} \bar{A}=d-1 \geq 1$, $e(\bar{A})=e(A)$ and $I(\bar{A}) \leq I(A)$. By induction we may assume that

$$
\ell\left(\bar{A} / \overline{\mathfrak{q}}^{i}\right) \leq e(A)\left(\begin{array}{c}
i+d-2 \\
d-1
\end{array}\right)+I(A)\left(\begin{array}{c}
i+d-3 \\
d-2
\end{array}\right)
$$

for all $i \geq 1$. From the exact sequence

$$
0 \longrightarrow \mathfrak{q}^{i-1}: x_{1} / \mathfrak{q}^{i-1} \longrightarrow A / \mathfrak{q}^{i-1} \stackrel{x_{1}}{\longrightarrow} A / \mathfrak{q}^{i} \longrightarrow \bar{A} / \overline{\mathfrak{q}}^{i} \longrightarrow 0
$$

we can deduce that

$$
\ell\left(\mathfrak{q}^{i-1} / \mathfrak{q}^{i}\right)=\ell\left(A / \mathfrak{q}^{i}\right)-\ell\left(A / \mathfrak{q}^{i-1}\right) \leq \ell\left(\bar{A} / \overline{\mathfrak{q}}^{i}\right)
$$

Using these formulas for $i=1, \ldots, n-1$, we get

$$
\begin{aligned}
\ell\left(A / \mathfrak{q}^{n}\right) & =\sum_{i=1}^{n} \ell\left(\mathfrak{q}^{i-1} / \mathfrak{q}^{i}\right) \leq \sum_{i=1}^{n-1} \ell\left(\bar{A} / \overline{\mathfrak{q}}^{i}\right) \\
& \leq \sum_{i=1}^{n}\left[e(A)\left(\begin{array}{c}
i+d-2 \\
d-1
\end{array}\right)+I(A)\left(\begin{array}{c}
i+d-3 \\
d-2
\end{array}\right)\right] \\
& =e(A)\left(\begin{array}{c}
n+d-1 \\
d
\end{array}\right)+I(A)\left(\begin{array}{c}
n+d-2 \\
d-1
\end{array}\right) .
\end{aligned}
$$

Remark. From earlier results on the Hilbert functions of local rings Tru1, Corollary 2.2], [DGV, Theorem 4.6], RVV Theorem 2.2] one can only derive the bound

$$
\ell\left(A / \mathfrak{m}^{n+1}\right) \leq D(A)\left(\begin{array}{c}
n+d-1 \\
d
\end{array}\right)+\left(\begin{array}{c}
n+d-1 \\
d-1
\end{array}\right) .
$$

This bound is much weaker than the bound of Theorem 2.1

We will use the above uniform bound for the Hilbert-Samuel function to give a local version of Mumford's Theorem 1.4. For this we shall need the following observations.

Let $G$ denote the associated graded ring $\bigoplus_{n>0} \mathfrak{m}^{n} / \mathfrak{m}^{n+1}$ of $A$. Let $x$ be a generic element of $\mathfrak{m}$. Without restriction we may assume that the initial form $x^{*}$ of $x$ in $G$ is a filter-regular element. Let $\bar{G}$ denote the associated graded ring of $A /(x)$.

Lemma 2.2 (cf. [ST2, Lemma 1]). g-reg $\left(G /\left(x^{*}\right)\right)=$ g-reg $(\bar{G})$.

Proof. We have

$$
\begin{gathered}
G /\left(x^{*}\right)=\bigoplus_{n \geq 0} \mathfrak{m}^{n} /\left(\mathfrak{m}^{n+1}+x \mathfrak{m}^{n-1}\right), \\
\bar{G}=\bigoplus_{n \geq 0}\left(\mathfrak{m}^{n}+(x)\right) /\left(\mathfrak{m}^{n+1}+(x)\right)=\bigoplus_{n \geq 0} \mathfrak{m}^{n} /\left(\mathfrak{m}^{n+1}+(x) \cap \mathfrak{m}^{n}\right) .
\end{gathered}
$$

Hence there is a natural graded epimorphism from $G /\left(x^{*}\right)$ to $\bar{G}$ whose kernel is

$$
E=\bigoplus_{n \geq 0}\left(\mathfrak{m}^{n+1}+(x) \cap \mathfrak{m}^{n}\right) /\left(\mathfrak{m}^{n+1}+x \mathfrak{m}^{n-1}\right) .
$$


Since $x$ is a superficial element, $\mathfrak{m}^{n+1}: x=\mathfrak{m}^{n}+(0: x)$ for all large $n$ N, Theorem (22.6)]. Thus, $(x) \cap \mathfrak{m}^{n+1}=x \mathfrak{m}^{n}$, so that $E_{n}=0$ for all large $n$. This implies $H_{G_{+}}^{i}\left(G /\left(x^{*}\right)\right)=H_{G_{+}}^{i}(\bar{G})$ for $i>0$. Hence g-reg $\left(G /\left(x^{*}\right)\right)=\operatorname{g}$-reg $(\bar{G})$.

For $n \geq \operatorname{reg}(\bar{G})$, we can bound $p_{G}(n)$ by means of the Hilbert-Samuel function of $A /(x)$. Note that $\operatorname{reg}(\bar{G}) \geq \mathrm{g}-\operatorname{reg}(\bar{G})$.

Lemma 2.3. $p_{G}(n)=\ell\left(A / \mathfrak{m}^{n+1}+(x)\right)-\ell(0: x)$ for $n \geq \operatorname{reg}(\bar{G})$.

Proof. By [N, Theorem (22.6)] we have

$$
h_{G}(n)=\ell\left(\mathfrak{m}^{n} / \mathfrak{m}^{n+1}\right)=\ell\left(A / \mathfrak{m}^{n+1}+(x)\right)-\ell(0: x)
$$

for all large $n$. Write $\ell\left(A / \mathfrak{m}^{n+1}+(x)\right)=\sum_{i=0}^{n} h_{\bar{G}}(i)$. By Lemma $1.3 h_{\bar{G}}(i)=p_{\bar{G}}(i)$ for $i>\operatorname{reg}(\bar{G})$. Hence $\ell\left(A / \mathfrak{m}^{n+1}+(x)\right)-\ell(0: x)$ is a polynomial function for $n \geq \operatorname{reg}(\bar{G})$. This polynomial must be $p_{G}(n)$.

Now we can deduce from Mumford's Theorem 1.4 the following local version.

Theorem 2.4. Assume that $d=\operatorname{dim} A \geq 2$. Let $I(A)$ be an arbitrary CohenMacaulay deviation of $A$. For $n \geq \operatorname{reg}(\bar{G})$ we have

$$
\text { g-reg }(G) \leq e(A)\left(\begin{array}{c}
n+d-2 \\
d-1
\end{array}\right)+I(A)\left(\begin{array}{c}
n+d-3 \\
d-2
\end{array}\right)
$$

Proof. By Theorem 1.4 and Lemma 2.2 we have

$$
\text { g-reg }(G) \leq n+p_{G}(n)-h_{G / L}(n)
$$

where $L$ is the largest ideal of finite length of $G$. Since $\operatorname{dim} G / L=d$,

$$
h_{G / L}(n) \geq\left(\begin{array}{c}
n+d-1 \\
d-1
\end{array}\right) .
$$

By Lemma 2.3 and Theorem 2.1,

$$
\begin{aligned}
p_{G}(n) & \leq \ell\left(A / \mathfrak{m}^{n+1}+(x)\right) \\
& \leq e(A /(x))\left(\begin{array}{c}
n+d-2 \\
d-1
\end{array}\right)+I(A /(x))\left(\begin{array}{c}
n+d-3 \\
d-2
\end{array}\right)+\left(\begin{array}{c}
n+d-2 \\
d-2
\end{array}\right) .
\end{aligned}
$$

Since $e(A /(x))=e(A)$ and $I(A /(x)) \leq I(A)$, we finally obtain

$$
\begin{aligned}
\operatorname{g}-\operatorname{reg}(G) \leq & n+e(A)\left(\begin{array}{c}
n+d-2 \\
d-1
\end{array}\right)+I(A)\left(\begin{array}{c}
n+d-3 \\
d-2
\end{array}\right) \\
& +\left(\begin{array}{c}
n+d-2 \\
d-2
\end{array}\right)-\left(\begin{array}{c}
n+d-1 \\
d-1
\end{array}\right) \\
\leq & e(A)\left(\begin{array}{c}
n+d-2 \\
d-1
\end{array}\right)+I(A)\left(\begin{array}{c}
n+d-3 \\
d-2
\end{array}\right) .
\end{aligned}
$$

\section{BOUNDS FOR THE REGULARITY}

Let $(A, \mathfrak{m})$ be a local ring with infinite residue field and $d=\operatorname{dim} A$. Let $G$ be the associated graded ring of $A$. We will apply Theorem 2.4 to compute $\operatorname{reg}(G)$. For that we shall need the following two propositions. The first proposition allows us to pass to the case depth $A>0$, while the second proposition shows that regularity coincides with geometric regularity in this case. 
Proposition 3.1. Let $G^{\prime}$ denote the associated graded ring of $A / L$, where $L$ is the largest ideal of $A$ of finite length. Then

$$
\operatorname{reg}(G) \leq \operatorname{reg}\left(G^{\prime}\right)+\ell(L) .
$$

Proof. We have

$$
G^{\prime}=\bigoplus_{n \geq 0}\left(\mathfrak{m}^{n}+L\right) /\left(\mathfrak{m}^{n+1}+L\right) \cong \bigoplus_{n \geq 0} \mathfrak{m}^{n} /\left(\mathfrak{m}^{n+1}+\mathfrak{m}^{n} \cap L\right) .
$$

Therefore, there is a natural graded epimorphism from $G$ to $G^{\prime}$ with the kernel

$$
K=\bigoplus_{n \geq 0}\left(\mathfrak{m}^{n+1}+\mathfrak{m}^{n} \cap L\right) / \mathfrak{m}^{n+1} \cong \bigoplus_{n \geq 0} \mathfrak{m}^{n} \cap L / \mathfrak{m}^{n+1} \cap L .
$$

Note that

$$
\ell(K)=\sum_{n \geq 0} \ell\left(\mathfrak{m}^{n} \cap L / \mathfrak{m}^{n+1} \cap L\right)=\ell(L) .
$$

Then $K$ has finite length. This implies $H_{G_{+}}^{i}(G) \cong H_{G_{+}}^{i}\left(G^{\prime}\right)$ for $i>0$.

Put $a=\operatorname{reg}\left(G^{\prime}\right)$ and $\ell=\ell(K)$. Then there exists an index $m$ with $a \leq m \leq a+\ell$ such that $K_{m+1}=0$. Since $G^{\prime}$ is $m$-regular, $H_{G_{+}}^{i}(G)_{m-i+1}=H_{G_{+}}^{i}\left(G^{\prime}\right)_{m-i+1}=0$ for $i \geq 0$. Hence $G$ is weakly $m$-regular. By Corollary [1.2, $G$ is $m$-regular, so that $\operatorname{reg}(G) \leq m \leq a+\ell$.

Proposition 3.2. Assume that $\operatorname{depth} A>0$. Then

$$
\operatorname{reg}(G)=\operatorname{g}-\operatorname{reg}(G) .
$$

Proof. Note that the result is trivial if $\operatorname{depth} G>0$. For any integer $i$ we set $a_{i}(G):=\max \left\{n \mid H_{G_{+}}^{i}(G)_{n} \neq 0\right\}$, where $a_{i}(M)=-\infty$ if $H_{G_{+}}^{i}(G)=0$. Then

$$
\begin{aligned}
\operatorname{reg}(G) & =\max \left\{a_{i}(G)+i \mid i \geq 0\right\}, \\
\mathrm{g}-\operatorname{reg}(G) & =\max \left\{a_{i}(G)+i \mid i>0\right\} .
\end{aligned}
$$

By [H, Theorem 5.2] (see also [Ma, Theorem 2.1]), the assumption $\operatorname{depth} A>0$ implies $a_{0}(G) \leq a_{1}(G)$. Hence $\operatorname{reg}(G)=\mathrm{g}$-reg $(G)$.

Now we are able to give an upper bound for the Castelnuovo-Mumford regularity of the associated graded ring in terms of any extended degree.

Theorem 3.3. Let $I(A)$ be an arbitrary Cohen-Macaulay deviation of $A$. Then

(i) $\operatorname{reg}(G) \leq e(A)+I(A)-1$ if $d=1$,

(ii) $\operatorname{reg}(G) \leq e(A)^{(d-1) !-1}\left[e(A)^{2}+e(A) I(A)+2 I(A)-e(A)\right]^{(d-1) !}-I(A)$ if $d \geq 2$.

Proof. Let $G^{\prime}$ and $L$ be as in Proposition 3.1. Then $\operatorname{reg}(G) \leq \operatorname{reg}\left(G^{\prime}\right)+\ell(L)$. Since $e(A)=e(A / L)$ and $I(A)=I(A / L)+\ell(L)$, we only need to prove the conclusion for the ring $A / L$. Replacing $A$ by $A / L$, we may assume that $\operatorname{depth} A>0$.

If $d=1$, then $A$ is a Cohen-Macaulay ring. Hence $I(A)=0$. By [Tru2, Theorem 5.1(i) and Theorem 1.2(iii)] we already know that $\operatorname{reg}(G) \leq e(A)-1$ (see also the proof of [ST1 Lemma 5]).

For $d \geq 2$ we choose a generic element $x$ of $\mathfrak{m}$ such that the initial form $x^{*}$ of $x$ is a filter-regular linear form in $G$. Note that $e(A /(x))=e(A)$ and $I(A /(x)) \leq I(A)$. 
Let $\bar{G}$ denote the associated graded ring of $A / x A$. Put $m=\operatorname{reg}(\bar{G})$. By Proposition 3.2 and Theorem 2.4 we have

$$
\operatorname{reg}(G)=\operatorname{g}-\operatorname{reg}(G) \leq e(A)\left(\begin{array}{c}
m+d-2 \\
d-1
\end{array}\right)+I(A)\left(\begin{array}{c}
m+d-3 \\
d-2
\end{array}\right) .
$$

Since

$$
\left(\begin{array}{c}
m+d-2 \\
d-1
\end{array}\right) \leq m^{d-1}, \quad\left(\begin{array}{c}
m+d-3 \\
d-2
\end{array}\right) \leq m^{d-2}
$$

this implies that

$$
\operatorname{reg}(G) \leq e(A) m^{d-1}+I(A) m^{d-2}
$$

If $d=2$, we have

$$
m \leq e(A / x A)+I(A / x A)-1 \leq e(A)+I(A)-1 .
$$

From this it follows that

$$
\begin{aligned}
\operatorname{reg}(G) \leq e(A) m+I(A) & =e(A)[e(A)+I(A)-1]+I(A) \\
& =\left[e(A)^{2}+e(A) I(A)+2 I(A)-e(A)\right]-I(A) .
\end{aligned}
$$

If $d \geq 3$, we have

$$
e(A) m^{d-1}+I(A) m^{d-2} \leq e(A)[m+I(A)]^{d-1}-I(A) .
$$

Using induction, we may assume that

$$
m \leq e(A)^{(d-2) !-1}\left[e(A)^{2}+e(A) I(A)+2 I(A)-e(A)\right]^{(d-2) !}-I(A) .
$$

Therefore, the last bound for $\operatorname{reg}(G)$ implies

$$
\begin{aligned}
\operatorname{reg}(G) & \leq e(A)\left\{e(A)^{(d-2) !-1}\left[e(A)^{2}+e(A) I(A)+2 I(A)-e(A)\right]^{(d-2) !}\right\}^{d-1}-I(A) \\
& \leq e(A)^{(d-1) !-1}\left[e(A)^{2}+e(A) I(A)+2 I(A)-e(A)\right]^{(d-1) !}-I(A) .
\end{aligned}
$$

The proof of Theorem 3.3 is now complete.

Corollary 3.4. Let $A$ be a Cohen-Macaulay local ring with $d=\operatorname{dim} A \geq 1$. Then

(i) $\operatorname{reg}(G) \leq e(A)-1$ if $d=1$,

(ii) $\operatorname{reg}(G) \leq e(A)^{2((d-1) !)-1}[e(A)-1]^{(d-1) !}$ if $d \geq 2$.

Proof. The fact that $A$ is Cohen-Macaulay implies $I(A)=0$. Hence the conclusion follows from Theorem 3.3

As one can see from the proof, the bound of Theorem 3.3 can be further improved if $d \geq 3$, but the formula is not so compact.

\section{Finiteness of Hilbert functions}

Let $(A, \mathfrak{m})$ be a local ring with infinite residue field and $d=\operatorname{dim} A$. We can bound the coefficients $e_{i}(A)$ in terms of any extended degree as follows.

Theorem 4.1. Let $I(A)$ be an arbitrary Cohen-Macaulay deviation of A. Then

(i) $\left|e_{1}(A)\right| \leq \frac{e(A)[e(A)-1]}{2}+I(A)$,

(ii) $\left|e_{i}(A)\right| \leq e(A)^{i !-i}\left[e(A)^{2}+e(A) I(A)+2 I(A)\right]^{i !}-1$ if $i \geq 2$. 
Proof. Let $L$ be the largest ideal of finite length of $A$. Note that

$$
\begin{aligned}
\ell\left(A / \mathfrak{m}^{n+1}\right) & =\ell\left(A / \mathfrak{m}^{n+1}+L\right)+\ell\left(\mathfrak{m}^{n+1}+L / \mathfrak{m}^{n+1}\right) \\
& =\ell\left(A / \mathfrak{m}^{n+1}+L\right)+\ell\left(L / \mathfrak{m}^{n+1} \cap L\right)=\ell\left(A / \mathfrak{m}^{n+1}+L\right)+\ell(L)
\end{aligned}
$$

for $n$ large. Then we have

$$
\begin{aligned}
e_{i}(A) & =e_{i}(A / L), i=0, \ldots, d-1, \\
\left|e_{d}(A)\right| & \leq\left|e_{d}(A / L)\right|+\ell(L) .
\end{aligned}
$$

If $d=1$, then $A / L$ is a Cohen-Macaulay ring. It is easy to see (see e.g. [Ki]) that

$$
\left|e_{1}(A / L)\right| \leq \frac{e(A / L)[e(A / L)-1]}{2} .
$$

Since $e(A)=e(A / L)$ and $I(A)=\ell(L)$, we get

$$
\left|e_{1}(A)\right| \leq\left|e_{1}(A / L)\right|+\ell(L) \leq \frac{e(A)[e(A)-1]}{2}+I(A) .
$$

If $d \geq 2$, we first consider the case $\operatorname{depth} A>0$. Choose a generic element $x$ of $\mathfrak{m}$ such that the initial form $z$ of $x$ is a filter-regular linear form in $G$. Since $x$ is a superficial element of $\mathfrak{m}$, we have $\operatorname{dim} A / x A=d-1, e_{i}(A)=e_{i}(A / x)$ for $i=$ $0, \ldots, d-1$ (see e.g. [N] Theorem (22.6)] or [ST2, Lemma 1]) and $I(A /(x)) \leq I(A)$. Using the induction hypothesis on $A /(x)$, we may assume that

$$
\begin{aligned}
&\left|e_{1}(A)\right| \leq \frac{e(A)[e(A)-1]}{2}+I(A), \\
&\left|e_{i}(A)\right| \leq e(A)^{i !-i}\left[e(A)^{2}+e(A) I(A)+2 I(A)\right]^{i !}-1, i=2, \ldots, d-1 .
\end{aligned}
$$

It remains to prove the bound for $e_{d}(A)$. We have

$$
(-1)^{d} e_{d}(A)=P_{A}(n)-\sum_{i=0}^{d-1}(-1)^{i} e_{i}(A)\left(\begin{array}{c}
m+d-i \\
d-i
\end{array}\right)
$$

for all $n>0$. Let $G$ be the associated graded ring of $A$. Since $\ell\left(A / \mathfrak{m}^{n+1}\right)=$ $\sum_{i=0}^{n} h_{G}(i)$, Lemma 1.3 yields $P_{A}(n)=\ell\left(A / \mathfrak{m}^{n+1}\right)$ for $n \geq \operatorname{reg}(G)$. Put

$$
m=e(A)^{(d-1) !-1}\left[e(A)^{2}+e(A) I(A)+2 I(A)\right]^{(d-1) !}-1 .
$$

Then $m \geq \operatorname{reg}(G)$ by Theorem 3.3 Therefore, using Theorem 2.1 we have

$$
\begin{aligned}
\left|e_{d}(A)\right| \leq & I(A)\left(\begin{array}{c}
m+d-2 \\
d-1
\end{array}\right)+\left(\begin{array}{c}
m+d-1 \\
d-1
\end{array}\right)+\left(\begin{array}{c}
m+d-1 \\
d
\end{array}\right) e-\left(\begin{array}{c}
m+d \\
d
\end{array}\right) e \\
& +\sum_{i=1}^{d-1}\left|e_{i}(A)\right|\left(\begin{array}{c}
m+d-i \\
d-i
\end{array}\right) \\
\leq & I(A)\left(\begin{array}{c}
m+d-2 \\
d-1
\end{array}\right)+\left(\begin{array}{c}
m+d-1 \\
d-1
\end{array}\right)+\sum_{i=1}^{d-1}\left|e_{i}(A)\right|\left(\begin{array}{c}
m+d-i \\
d-i
\end{array}\right) .
\end{aligned}
$$

Note that

$$
\left(\begin{array}{c}
m+d-2 \\
d-1
\end{array}\right) \leq m^{d-1}, \quad\left(\begin{array}{c}
m+d-i \\
d-i
\end{array}\right) \leq(d-i+1) m^{d-i} .
$$

A numerical computation shows that

$$
\left|e_{1}(A)\right| \leq \frac{e(A)[e(A)-1]}{2}+I(A) \leq \frac{m-I(A)-d+1}{d} .
$$


Moreover,

$$
\left|e_{i}(A)\right| \leq m, i=2, \ldots, d-1 .
$$

Then we get

$$
\begin{aligned}
\left|e_{d}(A)\right| \leq & I(A) m^{d-1}+d m^{d-1}+\frac{m-I(A)-d+1}{d} \cdot d m^{d-1} \\
& +\sum_{i=2}^{d-1} m^{d-i+1}(d-i+1) \\
= & m^{d}+d m^{d-1}+(d-2) m^{d-2}+\cdots+2 m^{2} \leq(m+1)^{d}-1 \\
= & e(A)^{d !-d}\left[e(A)^{2}+e(A) I(A)+2 I(A)\right]^{d !}-1 .
\end{aligned}
$$

Finally, we consider the case $\operatorname{depth} A=0$. As shown above, we have

$$
\begin{aligned}
& \left|e_{1}(A / L)\right| \leq \frac{e(A / L)[e(A / L)-1]}{2}+I(A / L), \\
& \left|e_{i}(A / L)\right| \leq e(A / L)^{i !-i}\left[e(A / L)^{2}+e(A / L) I(A / L)+2 I(A / L)\right]^{i !}-1, i=2, \ldots, d .
\end{aligned}
$$

Since $e(A)=e(A / L)$ and $I(A)=I(A / L)+\ell(L)$, this immediately implies

$$
\begin{gathered}
\left|e_{1}(A)\right|=\left|e_{1}(A / L)\right| \leq \frac{e(A)[e(A)-1]}{2}+I(A), \\
\left|e_{i}(A)\right|=\left|e_{i}(A / L)\right| \leq e(A)^{i !-i}\left[e(A)^{2}+e(A) I(A)+2 I(A)\right]^{i !}-1, i=2, \ldots, d-1, \\
\left|e_{d}(A)\right| \leq\left|e_{d}(A / L)\right|+\ell(L) \\
\leq e(A)^{d !-d}\left[e(A)^{2}+e(A) I(A / L)+2 I(A / L)\right]^{d !}-1+\ell(L) \\
\leq e(A)^{d !-d}\left[e(A)^{2}+e(A) I(A)+2 I(A)\right]^{d !}-1 .
\end{gathered}
$$

The proof of Theorem 4.1 is now complete.

Corollary 4.2. Let $A$ be a Cohen-Macaulay ring. Then

(i) $\left|e_{1}(A)\right| \leq \frac{e(A)[e(A)-1]}{2}$ (see e.g. [Ki] $)$,

(ii) $\left|e_{i}(A)\right| \leq e(A)^{3(i !)-i}-1$ if $i \geq 2$.

Proof. This follows from the fact that $I(A)=0$ for a Cohen-Macaulay ring $A$.

Remark. If $A$ is a Cohen-Macaulay ring, Srinivas and Trivedi [ST2, Theorem 1] already gave the bound

$$
\left|e_{i}(A)\right| \leq\left(9 e(A)^{5}\right)^{i !}, i=1, \ldots, d .
$$

If $A$ is a generalized Cohen-Macaulay ring, this bound has been extended by Trivedi [Tri2, Theorem 8] to

$$
\left|e_{i}(A)\right| \leq\left[(3+c)^{2} e(A)^{5}\right]^{i !}, i=1, \ldots, d,
$$

for some invariant $c \geq 2 I(A)$, where $I(A)=\sum_{i=0}^{d-1}\left(\begin{array}{c}d-1 \\ i\end{array}\right) \ell\left(H_{\mathfrak{m}}^{i}(A)\right)$ is the CohenMacaulay deviation of the homological degree $\operatorname{hdeg}(A)$. These bounds are worse than the bounds of Theorem 4.1 and Corollary 4.2 .

As an application of our bounds for the Castelnuovo-Mumford regularity and the coefficients of the Hilbert-Samuel polynomial we obtain the finiteness of HilbertSamuel functions of local rings with given dimension and extended degree. 
Corollary 4.3. Given two positive integers $d$ and $q$, there exist only a finite number of Hilbert-Samuel functions for a local ring $A$ with $\operatorname{dim} A=d$ and $D(A) \leq q$.

Proof. By Lemma 1.3 it follows that $P_{A}(n)=\ell\left(A / \mathfrak{m}^{n+1}\right)$ for $n \geq \operatorname{reg}(G)$. By Theorem 2.1 there are only a finite number of possibilities for $\ell\left(A / \mathfrak{m}^{n+1}\right)$ for a fixed $n$. Hence the finiteness of the number of the possibilities for the function $\ell\left(A / \mathfrak{m}^{n+1}\right)$ follows from the finiteness of possibilities for $\operatorname{reg}(G)$ and for the polynomial $P_{A}(n)$. Hence the conclusion follows from Theorem 3.3 and Theorem 4.1 .

Obviously, Corollary 4.3 implies the finiteness of the number of Hilbert-Samuel functions of Cohen-Macaulay rings with given dimension and multiplicity [ST2] and of generalized Cohen-Macaulay rings with given dimension, multiplicity and lengths of local cohomology modules [Tri2]. Moreover, our results can be modified to cover the finiteness of the number of Hilbert-Samuel functions of graded algebras with given dimension and extended degree [RVV].

To illustrate the above results we consider the following simple example.

Example. Let $r \geq 1$ be any integer, and consider the one-dimensional local ring $A=k[[x, y]] /\left(x^{2}, x y^{r}\right)$. Then $A$ is a non-Cohen-Macaulay local ring with multiplicity $e(A)=1$ and Hilbert-Samuel function

$$
\ell\left(A / \mathfrak{m}^{n+1}\right)= \begin{cases}2 n+1 & \text { for } \quad n \leq r \\ n+r+1 & \text { for } \quad n>r .\end{cases}
$$

This shows that there may be infinite Hilbert-Samuel functions for local rings with given dimension and multiplicity (see [ST1, Section 4] for a similar example with two-dimensional local domains). Since $L=(x) /\left(x^{2}, x y^{r}\right), A$ is a generalized CohenMacaulay ring with $I(A)=\ell(L)=r$. Hence $D(A)=r+1$.

\section{REFERENCES}

[BM] D. Bayer and D. Mumford, What can be computed in algebraic geometry? in: D. Eisenbud and L. Robbiano (eds.), Computational Algebraic Geometry and Commutative Algebra, Proceedings, Cortona (1991), Cambridge University Press, 1993, 1-48. MR 95d:13032

$[\mathrm{BH}]$ W. Bruns and J. Herzog, Cohen-Macaulay rings, Cambridge, 1993. MR 95h:13020

[DGV] L. R. Doering, T. Gunston and W. Vasconcelos, Cohomological degrees and Hilbert functions of graded modules, Amer. J. Math. 120 (1998), 493-504. MR 99h:13019

[E] D. Eisenbud, Commutative Algebra with a View toward Algebraic Geometry, Springer, 1995. MR 97a:13001

[EG] D. Eisenbud and S. Goto, Linear free resolutions and minimal multiplicity, J. Algebra 88 (1984), 89-133. MR 85f:13023

[Ki] D. Kirby, The reduction number of a one-dimensional local ring, J. London Math. Soc. 10 (1975), 471-481. MR 52:388

[Kl] S. Kleiman, Théorie des intersections et théorème de Riemann-Roch, in: SGA 6, Lecture Notes in Math. 225, Springer, 1971. MR 50:7133

[H] Lê Tuân Hoa, Reduction numbers of equimultiple ideals, J. Pure Appl. Algebra 109 (1996), 111-126. MR 97e:13027

[Ma] T. Marley, The reduction number of an ideal and the local cohomology of the associated graded ring, Proc. Amer. Math. Soc. 117 (1993), 335-341. MR 93d:13029]

$[\mathrm{Mu}]$ D. Mumford, Lectures on curves on an algebraic surface, Princeton Univ. Press, Princeton, 1966. MR 35:187

[N] M. Nagata, Local rings, Interscience, New York, 1962. MR 27:5790

[RVV] M. E. Rossi, G. Valla, and W. Vasconcelos, Maximal Hilbert functions, Results in Math. 39 (2001) 99-114. MR 2001m:13020

[ST1] V. Srinivas and V. Trivedi, A finiteness theorem for the Hilbert functions of complete intersection local rings, Math. Z. 225 (1997), 543-558. MR 98i:13034 
[ST2] V. Srinivas and V. Trivedi, On the Hilbert function of a Cohen-Macaulay local ring, J. Algebraic Geom. 6 (1997), 733-751. MR 98i:13033

[Tri1] V. Trivedi, Hilbert functions, Castelnuovo-Mumford regularity and uniform Artin-Rees numbers, Manuscripta Math. 94 (1997), no. 4, 485-499. MR 99a:13007

[Tri2] V. Trivedi, Finiteness of Hilbert functions for generalized Cohen-Macaulay modules, Comm. Algebra 29 (2) (2001), 805-813. MR 2002e:13038

[Tru1] Ngô Viêt Trung, Absolutely superficial sequences, Math. Proc. Camb. Phil. Soc. 93 (1983), 35-47. MR 84i:13019

[Tru2] Ngô Viêt Trung, Reduction exponent and degree bound for the defining equations of graded rings, Proc. Amer. Math. Soc. 101 (1987), 229-236. MR 89i:13031

[V1] W. Vasconcelos, The homological degree of a module, Trans. Amer. Math. Soc. 350 (1998), no. 3, 1167-1179. MR 98i:13046

[V2] W. Vasconcelos, Cohomological degrees of graded modules. Six lectures on commutative algebra (Bellaterra, 1996), 345-392, Progr. Math. 166, Birkhäuser, Basel, 1998. MR 99j:13012

Dipartimento di Matematica, Università di Genova, Via Dodecaneso 35, 16132 Genova, ITALY

E-mail address: rossim@dima.unige.it

Institute of Mathematics, Box 631, Bò Hô, 10000 Hanoi, Vietnam

E-mail address: nvtrung@thevinh.ncst.ac.vn

Dipartimento di Matematica, Università di Genova, Via Dodecaneso 35, 16132 Genova, ITALY

E-mail address: valla@dima.unige.it 